EXTENDED REPORT

\title{
Penetration of moxifloxacin into the human aqueous humour after oral administration
}

\author{
G Kampougeris, A Antoniadou, E Kavouklis, Z Chryssouli, H Giamarellou
}

Br J Ophthalmol 2005;89:628-631. doi: 10.1136/bjo.2004.050054

See end of article for authors' affiliations .....................

Correspondence to: Mr G Kampougeris, Cardiff Eye Unit, University Hospital of Wales, Heath Park, Cardiff CFl4 4XW UK; gkampougeris@ yahoo.gr

Accepted for publication 1 October 2004
Aims: To determine the pharmacokinetics of moxifloxacin, a new generation fluoroquinolone, in the anterior chamber of the human uninflamed eye.

Methods: 35 patients undergoing cataract surgery received two doses of $400 \mathrm{mg}$ of oral moxifloxacin with a 12 hour interval and were divided into six groups. Moxifloxacin levels in aqueous humour and serum were determined by a microbiological agar well diffusion technique at 2, 4, 6, 8, 10, and 12 hours after the second dose in each group respectively.

Results: Mean moxifloxacin levels in the anterior chamber were 1.20 (SD 0.35$) \mu \mathrm{g} / \mathrm{ml}$ at the 2 hours group, $1.22(0.48) \mu \mathrm{g} / \mathrm{ml}$ at the 4 hours group, $1.20(0.45) \mu \mathrm{g} / \mathrm{ml}$ at the 6 hours group, $1.58(0.38) \mu \mathrm{g} /$ $\mathrm{ml}$ at the 8 hours group, $1.37(0.44) \mu \mathrm{g} / \mathrm{ml}$ at the 10 hours group, and $1.23(0.55) \mu \mathrm{g} / \mathrm{ml}$ at the 12 hours group. The mean ratio of aqueous to serum moxifloxacin level was $38 \%$.

Conclusion: Moxifloxacin penetrates well into the anterior chamber of the human uninflamed eye after oral administration, reaching early significant levels, which are maintained for at least 12 hours and are much higher than the $\mathrm{MIC}_{90}$ values of Gram positive and Gram negative pathogens commonly implicated in intraocular infections with the exceptions of fluoroquinolone resistant staphylococci, MRSA, and Pseudomonas aeruginosa.
B acterial endophthalmitis is one of the most serious complications following intraocular operations and penetrating ocular trauma. In the first setting the commonest micro-organisms involved are either Gram positive (Staphylococcus epidermidis, Staphylococcus aureus, streptococci, Propionibacterium acnes) or Gram negative (Pseudomonas aeruginosa, Haemophilus influenza, and Serratia marcescens) while in post-traumatic endophthalmitis there is significant involvement of Bacillus cereus and Staphylococcus aureus. ${ }^{1}$

Systemically administered antibiotics have to penetrate the existing blood-ocular barriers in order to reach therapeutic intraocular levels. Although there is evidence from studies in humans and rabbits that intraocular penetration of systemic antibiotics is higher in inflamed eyes,,$^{2-4}$ there are other facts complicating this issue. Most of the antibiotics used have to be administered intravenously at frequent intervals, thus requiring hospitalisation for many days and increasing patient discomfort. Emerging antibiotic resistance of bacterial endophthalmitis strains is another fact complicating prevention or treatment of intraocular imfections, ${ }^{56}$ because it compromises the need of an antibiotic to reach levels in the anterior chamber much higher than the minimal inhibitory concentrations (MICs) in order to eliminate the microorganisms potentially inoculated during surgery. Among previously studied systemic antibiotics, fluoroquinolones and, in particular ciprofloxacin, ofloxacin, and levofloxacin (all of which can be administered orally), have been found to have significant penetration into aqueous humour and less so into the vitreous body, with concentrations usually exceeding MICs of pathogens implicated in intraocular infections..$^{7-9}$ Ciprofloxacin and ofloxacin are second generation fluoroquinolones while levofloxacin comprises the third generation of these drugs.

The aim of our study was to determine the ocular penetration of moxifloxacin, a new fourth generation fluoroquinolone. We measured moxifloxacin levels in the anterior chamber of human uninflamed eyes after oral administration at different time intervals after the last dose, studying also the rate through time of antibiotic decay.

\section{PATIENTS AND METHODS}

Thirty five patients, scheduled to undergo routine cataract surgery in "Laiko" General Hospital of Athens, participated in this study. The study was approved by the hospital ethics committee and all patients gave written informed consent. None of the participants had any history or evidence of intraocular inflammation, hepatic or renal failure, or history of allergic reactions to fluoroquinolones. None was on any oral or topical antibiotic treatment for at least the preceding 2 weeks and none had a previous operation in the eye to be operated.

All patients received two $400 \mathrm{mg}$ doses of oral moxifloxacin (Avelox, Bayer) with a 12 hour interval between them. Patients were randomly allocated into six groups (groups 16) and anterior chamber samples were taken 2, 4, 6, 8, 10, and 12 hours after the second dose for each one of the groups, respectively. Each group comprised six patients except from group 3, which comprised five patients. Participants were scheduled to have either extracapsular cataract extraction or phacoemulsification according to the surgeon's preference. After peribulbar or retrobulbar anaesthesia and while the patient was prepared for the operation, $5 \mathrm{ml}$ of blood were obtained for serum level determination. Periocular skin was scrubbed with 5\% povidone-iodine solution and an adherent window drape was applied. After insertion of a speculum, a 27 gauge needle attached to a $1 \mathrm{ml}$ syringe was introduced into the anterior chamber through a partial thickness limbal incision and $0.1 \mathrm{ml}$ of aqueous humour was aspirated.

Blood samples were centrifuged immediately afterwards and serum and aqueous samples were stored at $-70^{\circ} \mathrm{C}$. They were kept frozen until the time of analysis, which was in all

Abbreviations: MIC, minimal inhibitory concentration; MRSA, methicillin resistant Staphylococcus aureus 
Table 1 Mean moxifloxacin levels with their standard deviations in serum and aqueous humour for all groups

\begin{tabular}{llll}
\hline & $\begin{array}{l}\text { Time from last } \\
\text { dose to } \\
\text { sampling } \\
\text { (hours) }\end{array}$ & $\begin{array}{l}\text { Average moxifloxacin } \\
\text { levels in serum (SD) } \\
(\mu \mathrm{g} / \mathrm{ml})\end{array}$ & $\begin{array}{l}\text { Average moxifloxacin } \\
\text { levels in aqueous (SD) } \\
(\mu \mathrm{g} / \mathrm{ml})\end{array}$ \\
\hline Group 1 (6 patients) & 2 & $4.46(1.62)$ & $1.2(0.35)$ \\
Group 2 (6 patients) & 4 & $4.86(2.95)$ & $1.22(0.48)$ \\
Group 3 (5 patients) & 6 & $3.30(1.21)$ & $1.20(0.45)$ \\
Group 4 (6 patients) & 8 & $2.79(1.03)$ & $1.58(0.38)$ \\
Group 5 (6 patients) & 10 & $2.77(1.23)$ & $1.37(0.44)$ \\
Group 6 (6 patients) & 12 & $2.29(1.42)$ & $1.23(0.55)$ \\
\hline
\end{tabular}

cases within 4 weeks of sampling. Antibiotic level assays were performed using a microbiological agar well diffusion technique ${ }^{10}$ using Mueller-Hinton agar test as medium and $B$ subtilis ATCC 6633 as indicator micro-organism. High performance liquid chromatography (HPLC) which is nowadays widely used as a method of choice to assay drug levels, was not available in our laboratory at the time of the study, but the two dimension microbiological agar well assay used (if well designed and performed) has been proved to be sensitive and in excellent agreement with other techniques (immunological, HPLC). ${ }^{10-12}$

\section{RESULTS}

Of the 35 participants in this study 14 were male and 21 female. Their age ranged from 50-87 years (mean 73 years). Common underlying systemic diseases included hypertension, diabetes mellitus, hyperlipidaemia, and cardiovascular diseases, all of them well controlled at the time of surgery. In all cases the indication for surgery was visually significant cataract. In only one case was cataract secondary (steroid induced in a patient with scleroderma). Five of the 35 patients had glaucoma that was controlled with only topical medication. Four patients with glaucoma received oral acetazolamide before the operation to lower intraocular pressure further, depending on preoperative measurements. The acetazolamide dose was $250 \mathrm{mg}$ administered orally approximately 2 hours before surgery. The administration of acetazolamide did not seem to affect the penetration of moxifloxacin into the aqueous humour in these patients (data not shown). Diabetes also did not affect the penetration

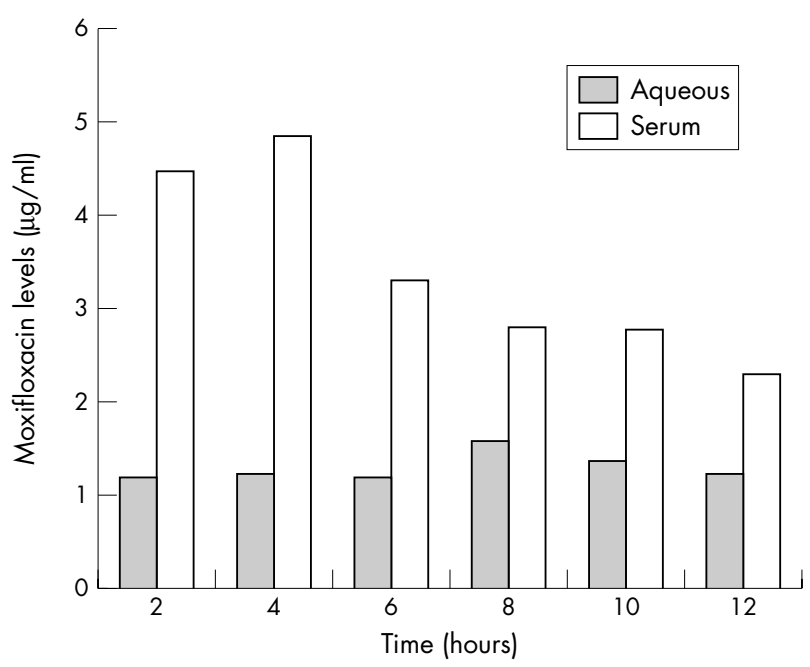

Figure 1 Mean moxifloxacin levels in aqueous and serum over time. of moxifloxacin and levels achieved were comparable between diabetic (10 patients) and not diabetic patients (data not shown).

There were no side effects from moxifloxacin administration in the patients enrolled in the study.

Moxifloxacin levels in aqueous and serum for all patient groups (expressed as mean values (SD)) are shown in table 1 . The average concentration of moxifloxacin in aqueous humour ranged between $1.2 \mu \mathrm{g} / \mathrm{ml}(2,4,6$, and 12 hours after the second dose) and $1.58 \mu \mathrm{g} / \mathrm{ml}$ ( 8 hours after the second dose). In serum, the average concentration ranged between $2.29 \mu \mathrm{g} / \mathrm{ml}$ ( 12 hours after the second dose) and $4.86 \mu \mathrm{g} / \mathrm{ml}$ (4 hours after the second dose). Peak concentrations were recorded at 4 hours after the second dose in serum and 8 hours after dosing in aqueous. Figure 1 depicts the mean antibiotic concentrations over time in aqueous and serum. From these data it is evident that moxifloxacin concentrations reach significant levels fairly soon ( 2 hours) after the last dose of the antibiotic, that remain high during the following hours, showing stability over time. Interpatient variation was greater for serum values than in aqueous humour values, where the results were clustered around the mean for all groups as shown in figure 2. Mean ratio of aqueous humour levels to serum levels was 38\%, ranging between 25\% (2-4 hours after dosing) and 57\% ( 8 hours after dosing).

\section{DISCUSSION}

Moxifloxacin is a new fourth generation fluoroquinolone with a broad spectrum of activity against Gram positive and

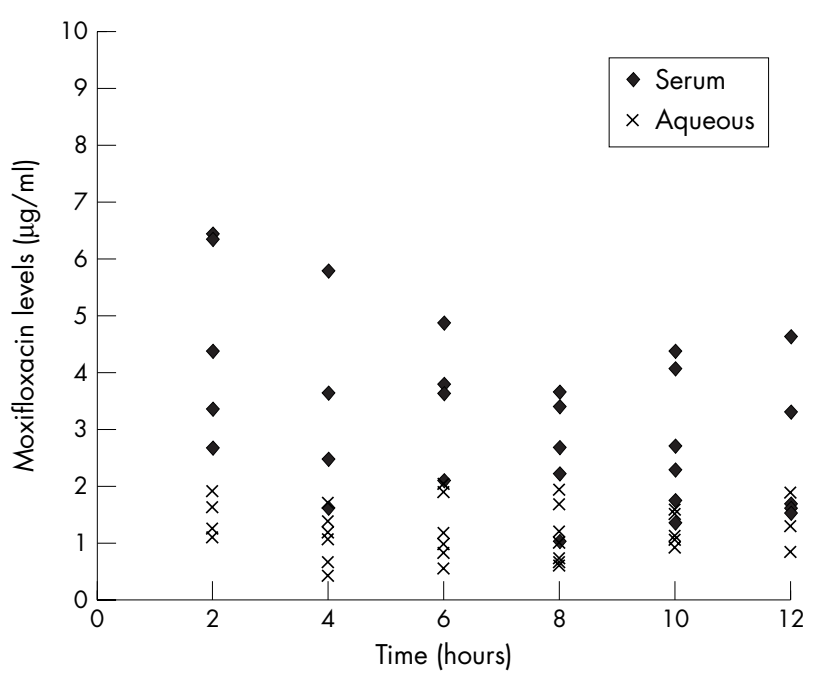

Figure 2 Distribution of moxifloxacin levels in serum and aqueous over time. 
Gram negative micro-organisms. In a recent study moxifloxacin was found to be the most potent fluoroquinolone for Gram positive bacteria (including methicillin resistant Staphylococcus aureus (MRSA) and ciprofloxacin resistant Staphylococcus aureus) and equally potent as other fluoroquinones against Gram negative bacteria, with the exception of $P$ aeruginosa against which ciprofloxacin remains the most potent fluoroquinolone. ${ }^{13}$ An advantage of moxifloxacin is its oral administration. Moxifloxacin displays linear pharmacokinetics in blood after a $400 \mathrm{mg}$ dose, with a half life of 8 hours (when administered orally or intravenously). It acts by dual targeting, inhibiting the action of DNA gyrase and topoisomerase IV, resulting in inhibition of bacterial replication and ultimate cell death. Because of this mode of action development of resistant strains among Gram positive cocci is limited, carrying a lower risk of cross resistance with other fluoroquinolones. ${ }^{14}{ }^{15}$ This is particularly important as resistant strains of pathogens to widely used fluoroquinolones in ophthalmology are reported. ${ }^{56}$ Moxifloxacin levels in the anterior chamber have been studied only once previously in a clinical study, where moxifloxacin levels were measured 10 hours after a single oral dose of $400 \mathrm{mg}$ in 15 patients. ${ }^{16}$ The mean aqueous levels at 10 hours were higher than in our study $(2.33 \mu \mathrm{g} / \mathrm{ml})$, though measured with a similar microbiological assay. Another recent study reported serum, aqueous, and vitreous levels of gatifloxacin, another new fourth generation fluoroquinolone (in clinical use in a limited number of countries), after oral administration. ${ }^{17}$ Mean levels of gatifloxacin in aqueous humour after two $400 \mathrm{mg}$ doses given 12 hours apart were $1.08(0.54) \mu \mathrm{g} / \mathrm{ml}$ (3.9 (1.1) hours after the last dose) and $1.34(0.34) \mu \mathrm{g} / \mathrm{ml}$ in vitreous (4 (1) hour after the last dose). These findings correlate well with results in our study (the aqueous humour levels) and confirm the very good penetration of new fourth generation fluoroquinolones in the human uninflamed eye. It also shows, for the first time in the case of systemically administered antibiotics, similar levels of gatifloxacin in aqueous humour and vitreous body, something that probably needs also to be investigated in the case of moxifloxacin.

This study is the first in the literature (to our knowledge) to access in an adequate number of patients the pharmacokinetics of orally administered moxifloxacin in the anterior chamber of the human, uninflamed eye. It showed that moxifloxacin penetrates well in the anterior chamber, reaching early significant levels soon after the second dose (at 2 hours) and remaining fairly stable for at least 12 hours. These levels were constantly much higher than the $\mathrm{MIC}_{90}$ of moxifloxacin against pathogens implicated in bacterial endophthalmitis with the exception of fluoroquinone resistant coagulase negative staphylococci, MRSA, and $P$ aeruginosa. ${ }^{18} 19$ These MIC $_{90}$ values were based on bacterial strains not isolated from endophthalmitis cases $^{18}{ }^{19}$; however the median MIC values for bacterial endophthalmitis isolates according to a recent study were comparable. ${ }^{13}$ In the later study moxifloxacin was the most potent among all fluoroquinolones tested (ciprofloxacin, ofloxacin, levofloxacin, and gatifloxacin). Moxifloxacin is especially very potent against Gram positive bacteria, which are the cause of the vast majority of cases of endophthalmitis. ${ }^{20}$ Median $\mathrm{MIC}_{90}$ values of moxifloxacin against common pathogens are shown in table 2, where it is evident that the drug exhibits excellent in vitro activity against an array of micro-organisms commonly encountered in bacterial endophthalmitis, except fluoroquinolone resistant Staph aureus. ${ }^{13}{ }^{21}$ Moxifloxacin levels in serum were in agreement with those of previous studies. ${ }^{14}$ The median ratio of aqueous to serum levels was 38\% and was significantly higher than the values obtained with other fluoroquinolones (15-23\% for ciprofloxacin, ${ }^{722} 23 \%$ for ofloxacin, ${ }^{23} 13-23 \%$ for levofloxacin ${ }^{9}$ and $21.02 \%$ for gatifloxacin ${ }^{17}$ depending on the total dose). This finding confirms that moxifloxacin has very good penetration in the aqueous humour through the intact blood-ocular barriers in the uninflamed eye.

The Endophthalmitis Vitrectomy Study showed no benefit of the use of systemic antibiotics in terms of final visual acuity in cases of endophthalmitis after cataract surgery or secondary lens implantation ${ }^{24}$ (antibiotics used were ceftazidime and amikacin). In any endophthalmitis case from any cause, as well as in prophylaxis of high risk eyes (for example, trauma), it would be advantageous for an antibiotic to reach such high levels intraocularly and for a sufficient duration of time in order to eliminate the most common pathogens encountered in these cases. New generation fluoroquinolones seem to be first line candidates for that

Table 2 The median minimum inhibitory concentrations $\left(\mathrm{MIC}_{90}\right)$ of common pathogens to moxifloxacin

\begin{tabular}{|c|c|c|}
\hline & $\begin{array}{l}\text { Isolated in bacterial } \\
\text { endophthalmitis cases } \\
\text { (peak aqueous levels/MIC } / \mathrm{Co}) \\
(\mu \mathrm{g} / \mathrm{ml})\end{array}$ & $\begin{array}{l}\text { Isolated in systemic } \\
\text { infections (peak aqueous } \\
\text { levels/MIC } / \mathrm{MI}_{90}(\mu \mathrm{g} / \mathrm{ml})\end{array}$ \\
\hline MSSA & & $0.12(13.3)$ \\
\hline MRSA & & 4 \\
\hline Staphylococcus aureus FQR & 1.75 & \\
\hline Staphylococcus aureus FQS & $0.06(26.6)$ & 0.06 \\
\hline CoagNeg staphylococcus FQR & 2.5 & 2 \\
\hline CoagNeg staphylococcus FQS & $0.05(32)$ & $0.13(12.3)$ \\
\hline Streptococcus pneumoniae & 0.09 (17.7) & 0.25 \\
\hline Streptococcus viridans & $0.13(12.3)$ & 0.25 \\
\hline Enterococcus species & $0.19(8.4)$ & 0.5 \\
\hline Gram negatives & $0.08(20)$ & $0.25(6.4)$ \\
\hline Bacillus species & 0.09 (17.7) & \\
\hline Escherichia coli & & $0.06(26.6)$ \\
\hline Neisseria species & & $0.015(106)$ \\
\hline$H$ influenzae & & $0.06(26.6)$ \\
\hline Pseudomonas aeruginosa & & 8 \\
\hline \multicolumn{3}{|c|}{$\begin{array}{l}\text { FQR, resistant to ciprofloxacin and ofloxacin as determined by disc diffusion; FQS, susceptible to ciprofloxacin and } \\
\text { ofloxacin as determined by disc diffusion; Gram negatives, assortment of various Gram negative bacteria isolated } \\
\text { from endophthalmitis cases (mainly Haemophilus sp and } P \text { aeruginosa) })^{13} ; \text { MSSA, methicillin sensitive } \\
\text { Staphylococcus aureus; MRSA, methicillin resistant Staphylococcus aureus. }\end{array}$} \\
\hline
\end{tabular}


and are favoured because of the convenience of oral administration (although not ideal drugs for the rare cases of pseudomonal infections of the eye).

Prevention of the devastating results of intraocular infections justifies the interest and research in this era. Moxifloxacin, because of its easy way of administration, relative safety compared to other fourth generation quinolones (less QT prolongation, no effect on glycaemic control), ${ }^{25} 26$ broad antimicrobial spectrum, and very good penetration into the anterior chamber and steady levels as shown in our study, could be a valuable addition to our armamentarium in the prevention and treatment of bacterial endophthalmitis, and warrants further clinical investigation.

\section{Authors' affiliations}

G Kampougeris, E Kavouklis, Department of Ophthalmology, Laiko General Hospital, Athens, Greece

A Antoniadou, Z Chryssouli, H Giamarellou, 4th Department of Internal Medicine, Athens University School of Medicine, Attikon University General Hospital, Athens, Greece

The authors have no proprietary interest in any of the antimicrobials mentioned in this manuscript.

\section{REFERENCES}

1 Marx JL. Endophthalmitis. In: Yanoff M, Duker JS, eds. Ophthalmology. London: Mosby, 1999:10.21.1-6.

2 Kanski JJ, Young JH, Vogel R. Penetration of intravenously administered cefoxitin into the aqueous humor of inflamed human eyes. Am J Ophthalmol 1982;94:516-18.

3 Aquilar HE, Meredith TA, Shaarawy A, et al. Vitreous cavity penetration of ceftazidime after intravenous administration. Retina 1995;15:154-9.

4 Alfaro DV, Hudson SJ, Rafanan MM, et al. The effect of trauma on the ocular penetration of intravenous ciprofloxacin. Am J Ophthalmol 1996;122:678-83.

5 Goldstein MH, Kowalski RP, Gordon YJ. Emerging fluoroquinolone resistance in bacterial keratitis: a 5-year review. Ophthalmology 1999;106:1313-18.

6 Gordon YJ. Vancomycin prophylaxis and emerging resistance: are ophthalmologists the villains? The heroes? Am J Ophthalmol 2001;131:371-6.

7 Lesk MR, Ammann H, Marcil G, et al. The penetration of oral ciprofloxacin into the aqueous humor, vitreous and subretinal fluid of humans. Am J Ophthalmol 1993;115:623-8

8 Donnenfeld ED, Perry HD, Snyder RW, et al. Intracorneal, aqueous humor and vitreous humor penetration of topical and oral ofloxacin. Arch Ophthalmol 1997;115:173-6.
9 Fiscella RG, Nguyen TK, Cwik MJ, et al. Aqueous and vitreous penetration of levofloxacin after oral administration. Ophthalmology 1999; 106:2286-90

10 Klassen M, Edberg SC. Measurement of antibiotics in human body fluids: techniques and significance. In: Lorian V, eds. Antibiotics in laboratory medicine. Baltimore: William and Wilkins, 1996:230-95.

11 Al-Meshal MA, Ramadan MA, Lotfi KM, et al. Determination of meropenem in plasma by high-performance liquid chromatography and a microbiological method. J Clin Pharm Ther 1995;20:158-63.

12 Wong PY, Zhu M, Li RC. Pharmacokinetic and pharmacodynamic interactions between intravenous ciprofloxacin and oral ferrous sulfate. J Chemother 2000;12:286-93

13 Mather R, Karenchak LM, Romanowski EG, et al. Fourth generation fluoroquinolones: new weapons in the arsenal of ophthalmic antibiotics. Am J Ophthalmol 2002; 133:463-66.

14 Schentag JJ, Gilliland KK, Paladino JA. What have we learned from pharmacokinetic and pharmacodynamic theories? Clin Infect Dis 2001;32(Suppl 1):S39-46.

15 Hooper D. Mechanisms of action of antimicrobials: focus on fluoroquinolones. Clin Infect Dis 2001;32(Suppl 1):S9-15.

16 Garcia-Saenz MC, Arias-Puente A, Fresnadillo-Martinez M, et al. Human aqueous humor levels of oral ciprofloxacin, levofloxacin and moxifloxacin. $J$ Cataract Refract Surg 2001;27:1969-74.

17 Hariprasad SM, Mieler WF, Holz ER. Vitreous and aqueous penetration of orally administered gatifloxacin in humans. Arch Ophthalmol 2003; 121:345-50.

18 Blondeau JM. A review of the comparative in-vitro activities of 12 antimicrobial agents, with a focus on five new "respiratory quinolones". J Antimicrob Chemother 1999;43(Suppl B):1-11.

19 Krasemann C, Meyer J, Tillotson G. Evaluation of the clinical microbiology profile of moxifloxacin. Clin Infect Dis 2001;32(Suppl 1):S51-63.

20 Han DP, Wisniewski SR, Wilson LA, et al. Spectrum and susceptibilities of microbiologic isolates in the Endophthalmitis Vitrectomy Study. Am J Ophthalmol 1996;122:1-17.

21 Andriole VT. Quinolones. In: Finch RG, Greenwood D, Norrby SR, Whitley RJ, eds. Antibiotics and chemotherapy. Philadelphia: Churchill-Livingstone, 2003, Chapter 29.

22 Morlet N, Graham GG, Gatus B, et al. Pharmacokinetics of ciprofloxacin in the human eye: a clinical study and population pharmacokinetic analysis. Antimicrob Agents Chemother 2000;44:1674-9.

23 Bron A, Talon D, Delbose B, et al. Intracameral penetration of ofloxacin in man. J Fr Ophtalmol 1987; 10:443-6.

24 Endophthalmitis Vitrectomy Study Group. Results of the endophthalmitis vitrectomy study. A randomised trial of immediate vitrectomy and of intravenous antibiotics for the treatment of postoperative bacterial endophthalmitis. Arch Ophthalmol 1995;113:1479-96.

25 Frothingham R. Rates of torsades de pointes associated with ciprofloxacin, ofloxacin, levofloxacin, gatifloxacin, and moxifloxacin. Pharmacotherapy $2001 ; 21: 1468-72$

26 Gavin JR, Kubin R, Choudri S, et al. Moxifloxacin and glucose homeostasis : a pooled-analysis of the evidence from clinical and postmarketing studies. Drug Saf 2004;27:671-86. 